\title{
Modifications of the electronic structure of GaSb surface by chalcogen atoms: S, Se, and Te
}

\author{
Z. Y. Liu, A. A. Gokhale, M. Mavrikakis, D. A. Saulys, and T. F. Kuech ${ }^{\text {a) }}$ \\ Department of Chemical and Biological Engineering, University of Wisconsin-Madison, \\ Madison, Wisconsin 53706
}

(Received 4 May 2004; accepted 19 July 2004)

\begin{abstract}
Modifications to the electronic properties and chemical structures of the GaSb surface using the chalcogen atoms $\mathrm{S}, \mathrm{Se}$, and Te were investigated theoretically and experimentally. A self-consistent density-functional theory study indicates that an adsorption of a full monolayer coverage of chalcogen atoms on a Ga-terminated surface reduces the density of gap region states significantly. A greater photoluminescence enhancement was observed from $\mathrm{GaSb}$ samples treated by chalcogenide $\left(\mathrm{Na}_{2} \mathrm{~S}, \mathrm{Na}_{2} \mathrm{Se}\right.$, or $\left.\mathrm{Na}_{2} \mathrm{Te}\right)$ in a nonaqueous than in an aqueous passivation medium. $\mathrm{X}$-ray photoelectron spectroscopy reveals a Ga-rich surface after a nonaqueous passivation, with sulfidization providing a higher concentration of $\mathrm{Ga}(\mathrm{Sb})$-chalcogen bonds than does a passivation with $\mathrm{Na}_{2} \mathrm{Se}$ or $\mathrm{Na}_{2} \mathrm{Te}$. The uptake of chalcogen during the passivation is accompanied by the loss of surface antimony. The formation of Sb- $X(X=\mathrm{S}$, Se, or Te) bonds competes with $X$ displacing surface $\mathrm{Sb}$, which dominates Se or Te incorporation in the GaSb surface lattice. The passivation kinetics was analyzed on basis of a single precursor-mediated coverage-dependent chemisorption proces. (C) 2004 American Institute of Physics. [DOI: 10.1063/1.1790572]
\end{abstract}

\section{INTRODUCTION}

$\mathrm{GaSb}$ is an important III-V compound semiconductor for high-speed and optoelectronic devices operating in the infrared and near-infrared region. ${ }^{1}$ However, the applications of $\mathrm{GaSb}$ in device structures are hindered by the complexities and difficulties in controlling the electronic structure of surfaces and interfaces. Air oxidation of GaSb leads to a thick overlayer of native oxide on the surface, along with a high concentration of elemental antimony. ${ }^{2-4}$ As for most other III-V semiconductors, the high density of energy states within the band-gap region results in a large surface band bending and reduces a minority carrier lifetime. It is important to control the electronic structure and state density of the $\mathrm{GaSb}$ surface to increased control, reliability, and performance of electronic devices.

The chemisorption of group VI elements on the GaAs surface can effectively reduce the surface-state density and surface recombination velocity, thus improving the optical and electrical performances of the GaAs-based devices. ${ }^{5,6}$ The remarkable enhancement in the photoluminescence (PL) yield from S-or Se-treated GaAs indicates that the chalcogenide-terminated GaAs surface exhibits an improved electronic surface structure relative to the native oxidized surface. ${ }^{7,8}$ In particular, Se-based treatments result in GaAs surfaces more chemically stable than those prepared by S-based aqueous treatments. Due to the similarity of the covalent radii of As and $\mathrm{Se}$, Se is easily incorporated into the GaAs lattice by replacement of the surface As. The formation of a stable $\mathrm{Ga}-\mathrm{Se}$ surface phase can reduce the structure-related defect density. ${ }^{9,10}$ A better understanding of the interactions between the passivation adsorbates and the

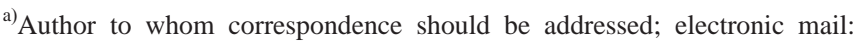
kuech@engr.wisc.edu
}

semiconductor surface will provide insights in improving the electronic passivation techniques for III-V semiconductors.

Sulfur has been the dominant element in most passivation studies. Few investigations on the use of selenium and tellurium have been reported, except for those involving GaAs. ${ }^{11-13}$ In this study, the chemical nature of the passivated GaSb surface and its impact on the surface electronic structure were determined, as the passivating adsorbates were systematically altered from $\mathrm{S}$ to $\mathrm{Se}$ to Te. Both theoretical and experimental results are presented in this work. A self-consistent study of electronic states was performed for both $\mathrm{Ga}$ - and $\mathrm{Sb}$-terminated $\mathrm{GaSb}(001)$ surfaces. The calculated results, based on the density-functional theory, were used to determine the variation in the density of states (DOS) of $\mathrm{GaSb}$ surface adsorbed by group VI elements $(\mathrm{O}, \mathrm{S}, \mathrm{Se}$, and $\mathrm{Te}$ ) up to a monolayer. Experimentally, the electronic passivation efficiency was evaluated by the PL yield emitted from the $\mathrm{GaSb}$ samples prepared by $\mathrm{S}, \mathrm{Se}$, and Te-based aqueous and nonaqueous solutions. In general, a consistent PL enhancement was observed after a nonaqueous passivation treatment with each of the chalcogenides. X-ray photoelectron spectroscopy (XPS) analysis reveals the chemical structure of the passivated surfaces. Finally, using $\mathrm{Na}_{2} \mathrm{Se}$ as a representative chalcogenide, the uptake of $\mathrm{Se}$ on the $\mathrm{GaSb}$ surface as a function of processing time was investigated, and the adsorption process was analyzed on the basis of the single precursor-mediated chemisorption.

\section{EXPERIMENTAL PROCEDURES}

A series of experiments was performed to compare the electronic passivation efficiency of S, Se, and Te. Samples of Te-doped $n$-type $\mathrm{GaSb}(001)$ with a carrier concentration of $\sim 10^{17} \mathrm{~cm}^{-3}$ were used for all the measurements. The nonaqueous (benzene) passivation solution consists of a sodium 
chalcogenide $\left(\mathrm{Na}_{2} \mathrm{~S}, \mathrm{Na}_{2} \mathrm{Se}\right.$, or $\left.\mathrm{Na}_{2} \mathrm{Te}\right)$, a macrocyclic polyether (15-crown-5), and an organic oxidizing agent (anthraquinone). The details of this process were described previously. ${ }^{14} \mathrm{GaSb}$ wafers were immersed in such passivation solutions for an hour at room temperature and rinsed with two propanol. For comparison, a second series of GaSb samples was exposed to the saturated chalcogenide aqueous solutions. A nonpassivated sample prepared by the chemical degreasing of an as-received $n$-GaSb substrate was used as a control for the photoluminescence measurement.

The PL measurements were performed at room temperature. The peak intensity, relative to that from the control sample, was recorded as a measure of the optical and electronic properties of the GaSb sample with a specific chalcogen treatment. The chemical properties of GaSb surface absorbed with chalcogen atoms, particularly in the benzenebased reaction medium, were investigated by XPS using $\mathrm{AlK}_{\alpha}$ radiation $(\mathrm{h} \nu=1486.6 \mathrm{eV})$ as an excitation source. The adsorption kinetics was studied using $\mathrm{Se}$ as a representative adsorbate. The $\mathrm{Se}$ uptake on the $\mathrm{GaSb}(001)$ surface was achieved by dipping the GaSb substrate in the benzene-based $\mathrm{Na}_{2} \mathrm{Se}$ solution with a certain time, varies from 5,20, and $40 \mathrm{~s}$, through 1, 5, 10, 20, and $60 \mathrm{~min}$ at room temperature. After each treatment, the atomic ratio of Se to Ga was then determined by comparing their specific XPS peak intensities.

The unsaturated surface bonds and structural defects can lead to energy states within the band gap of the bulk material. Adatoms, such as group VI elements, form chemical bonds with the semiconductor surface atoms and thus alter the distribution of surface states by removing the states from the midgap region. The total energy calculations based on the periodic self-consistent density-functional theory were performed on both Ga- and Sb-terminated $\mathrm{GaSb}(001)$ surfaces to elucidate the origin of surface states and the passivation mechanism with chalcogen atoms adsorption. The system was modeled by a $2 \times 2$ surface unit cell to study the adsorption of group VI elements. The unit contained four-layer slabs periodically repeated in a supercell geometry with five equivalent layers of vacuum between two successive slabs. The top two layers of the slab were relaxed to account for the surface relaxation effects. The surface Brillouin zone was sampled at $K$-point grids corresponding to $4 \times 4 K$-points in the primitive surface unit cell. The exchange-correlation energy and potential were described by the generalized gradient approximation (GGA-PW91). ${ }^{15,16}$ The total electronic state density of the $\mathrm{GaSb}(001)$ surface was monitored before and after the adsorption of group VI elements $(\mathrm{O}, \mathrm{S}, \mathrm{Se}$, and $\mathrm{Te})$ up to a monolayer.

\section{RESULTS}

Photoluminescence due to band-to-band transitions is modulated by the near surface electronic properties. In most cases, the photoluminescence yield can be simply related to the thickness of the surface depletion region and the surface recombination velocity. ${ }^{17,18}$ The variation of the PL intensity from $n$-GaSb after chalcogen treatments in aqueous and nonaqueous solutions are listed in Table I. The values are the average of multiple separately treated samples and are refer-
TABLE I. Room-temperature photoluminescence from $n$-GaSb samples after an hour chalcogenide treatments.

\begin{tabular}{lccc}
\hline \hline \multicolumn{1}{c}{ Solvents } & $\mathrm{Na}_{2} \mathrm{~S}$ & $\mathrm{Na}_{2} \mathrm{Se}$ & $\mathrm{Na}_{2} \mathrm{Te}$ \\
\hline (a) Water & $3.5 \pm 0.4$ & $5.8 \pm 0.6$ & $2.4 \pm 0.4$ \\
(b) Benzene based & $8.0 \pm 0.7$ & $8.2 \pm 0.6$ & $7.9 \pm 0.7$ \\
\hline
\end{tabular}

enced to the control sample. The variation in PL intensity is dependent on both the specific adsorbate and the particular passivation process. The GaSb samples prepared by using benzene-based nonaqueous solutions exhibited a large PL enhancement, up to an eight fold increase relative to the nonpassivated reference sample. Also, the luminescence yields were comparable for each of the three chalcogens applied. In contrast, results from the samples prepared by aqueous solutions indicated that the passivation by $\mathrm{Na}_{2} \mathrm{Se}$ led to a higher PL intensity (5.8) than those by $\mathrm{Na}_{2} \mathrm{~S}(3.5)$ or $\mathrm{Na}_{2} \mathrm{Te}(2.4)$.

The surface chemical properties were obtained by the XPS analysis. The gallium and antimony-related surface bonding states were determined after each nonaqueous chalcogen treatment, and representative spectra are presented in Fig. 1. The surface atomic composition was also estimated and listed in Table II with the estimated errors within the range of \pm 0.04 . The chemisorption of chalcogen atoms on the GaSb surface is accompanied by a decrease in both the amounts of native surface oxide and the $\mathrm{Sb}: \mathrm{Ga}$ ratio. The overall $\mathrm{Sb} / \mathrm{Ga}$ peak intensity ratio is $\sim 0.56$ after the $\mathrm{S}$ treatment, but decreases to $\sim 0.44$ after the $\mathrm{Se}$ - or Te-based treatments. In general, sulfidization yields a higher concentration of $\mathrm{Ga}(\mathrm{Sb})$-chalcogen species than those by the passivation with $\mathrm{Na}_{2} \mathrm{Se}$ or $\mathrm{Na}_{2} \mathrm{Te}$. In particular, the surface concentration

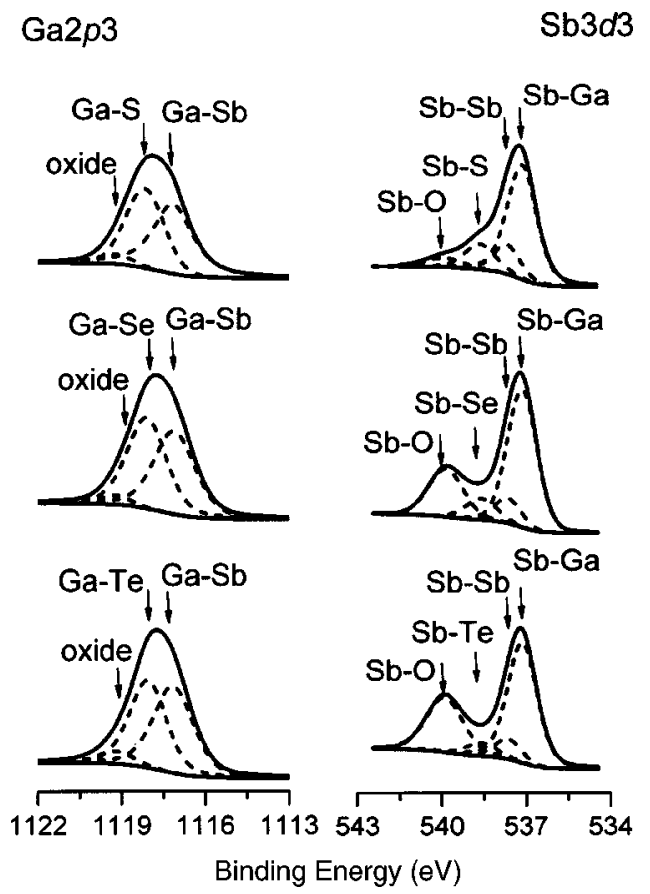

FIG. 1. Ga2p3 and Sb3d3 XPS spectra recorded from $n$-GaSb surface after chemical passivation treatments in the nonaqueous reaction medium. The chalcogenide sources used are $\mathrm{Na}_{2} \mathrm{~S}, \mathrm{Na}_{2} \mathrm{Se}$, and $\mathrm{Na}_{2} \mathrm{Te}$. The nonaqueous solutions were made from anhydrous benzene, with the addition of 15crown-5, an electron acceptor, and anthraquinone. 
TABLE II. Surface chemical composition of $n$-GaSb after $\mathrm{Na}_{2} \mathrm{~S}, \mathrm{Na}_{2} \mathrm{~S}$, or $\mathrm{Na}_{2} \mathrm{Te}$ nonaqueous treatments $(X=\mathrm{S}, \mathrm{Se}$, or Te). The values were estimated based on the XPS analysis, and the errors are within \pm 0.04 .

\begin{tabular}{lccc}
\hline \hline Treatments & $\mathrm{Na}_{2} \mathrm{~S}$ & $\mathrm{Na}_{2} \mathrm{Se}$ & $\mathrm{Na}_{2} \mathrm{Te}$ \\
\hline $\mathrm{Sb} / \mathrm{Ga}$ (overall peak intensity) & 0.56 & 0.44 & 0.46 \\
$\mathrm{Ga}-X /(\mathrm{Ga}+\mathrm{Sb})$ & 0.37 & 0.33 & 0.29 \\
$\mathrm{Ga}-\mathrm{O} /(\mathrm{Ga}+\mathrm{Sb})$ & 0.04 & 0.06 & 0.10 \\
$\mathrm{Sb}-\mathrm{Sb} /(\mathrm{Ga}+\mathrm{Sb})$ & 0.08 & 0.05 & 0.05 \\
$\mathrm{Sb}-X /(\mathrm{Ga}+\mathrm{Sb})$ & 0.11 & 0.05 & 0.04 \\
$\mathrm{Sb}-\mathrm{O} /(\mathrm{Ga}+\mathrm{Sb})$ & 0.04 & 0.13 & 0.18 \\
\hline \hline
\end{tabular}

of $\mathrm{Sb}-\mathrm{Se}$ or $\mathrm{Sb}-\mathrm{Te}$ is much lower than that of $\mathrm{Sb}-\mathrm{S}$; instead a large amount of antimony (native) oxide remains after the treatment with the heavier chalcogenides, Se and Te.

To elucidate the origin of surface states and the passivation mechanism by chalcogen atom adsorption, the electronic properties of $\mathrm{Ga}$ - and Sb-terminated $\mathrm{GaSb}(001)$ surfaces before and after adsorbing a monolayer of group VI elements $(\mathrm{O}, \mathrm{S}, \mathrm{Se}$, and $\mathrm{Te})$ were explored and the total DOS distributions are shown in Fig. 2. On the clean GaSb(001) surface, a continuous distribution of surface states across the entire $\mathrm{GaSb}$ energy-gap region was found for both the Ga- and Sb-terminated surfaces. Adsorption of a full monolayer coverage of oxygen on the Ga-terminated surface reduces the gap-region DOS significantly, but reduces the Sb-terminated surface-state density only slightly. Similar trends are exhibited with the chemisorption of $\mathrm{S}, \mathrm{Se}$, and $\mathrm{Te}$ on the $\mathrm{GaSb}(001)$ surface.
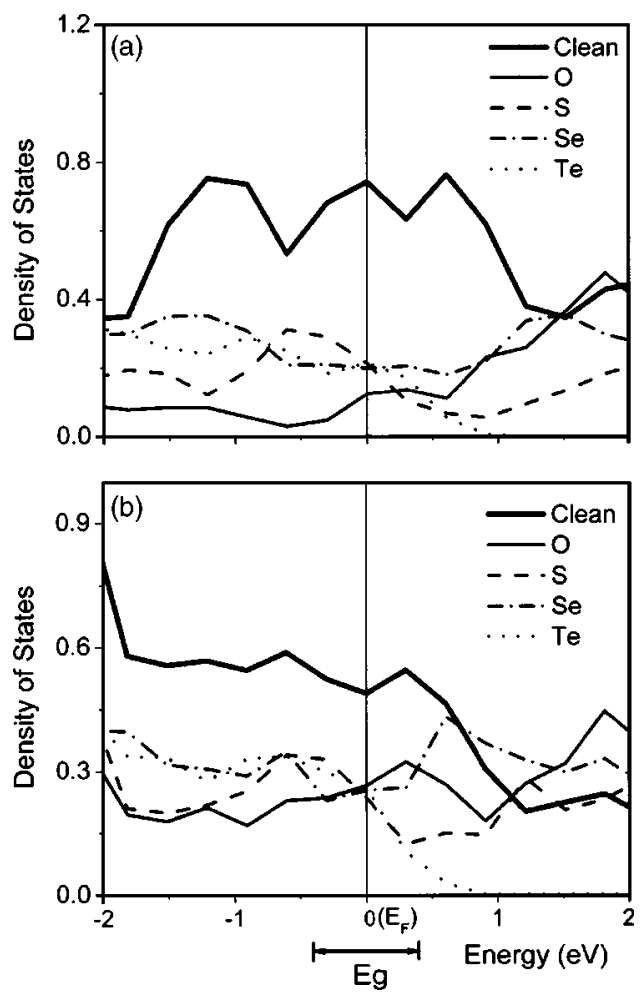

FIG. 2. Total density of states of (a) Ga- and (b) Sb-terminated GaSb(001) surfaces before and after adsorption of a monolayer of group VI atoms. The indicated energy gap $E_{g}$ represents the band gap of bulk GaSb, and the zero energy is defined as the Fermi level $\left(E_{F}\right)$, which is in the middle of the band gap for intrinsic GaSb.

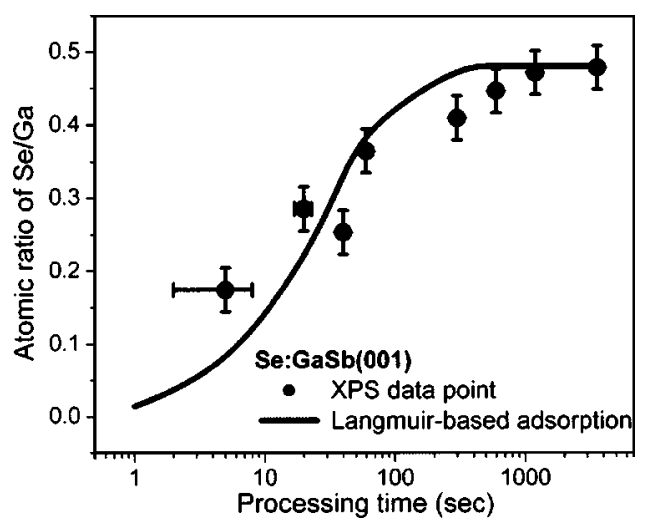

FIG. 3. Evolution of the surface selenide content vs the processing time. The solid dots are the atomic ratio of Se-to-Ga, derived from the XPS peak intensity ratio of Se and $\mathrm{Ga}$, with each processing time. The line is the least square fit of the kinetic data to the Langmuir-based adsorption mode in Eq. (5) at a $95 \%$ confidence limit.

The uptake of Se on the $\mathrm{GaSb}(001)$ surface was investigated by XPS. The Se and Ga photoemission spectra were recorded after each processing time, and the evolution of Se-to-Ga peak intensity ratio as a function of processing time is shown in Fig. 3. The initial uptake of $\mathrm{Se}$ on the $\mathrm{GaSb}$ surface proceeds rapidly. Subsequent exposure results in a continuous increase in Se content on the surface but at a much lower rate.

\section{DISCUSSION}

\section{A. Surface Electronic and Chemical Properties}

Surface states are those energy levels localized near the surface region of a semiconductor. Such electronic states lying within the band gap of a bulk semiconductor could be generated due to the unsaturated surface bonds, surface impurities, or structural defects. An ideal (001) plane of a III-V semiconductor is terminated by cations or anions, and each surface atom possesses two dangling bonds. A surface with unsaturated bonds is unstable and tends to reconstruct to reduce the surface energy. Adatoms, such as group VI elements, form chemical bonds with semiconductor surface atoms, thus altering the surface electronic structure. In most cases, the bonding configuration of an adatom-covered surface is different from the bulk structure. The deformation of the bond angle and the variation of the bond length result in a local strain, thus increasing the total energy of the surface. The combined electronic and structural effects are important in determining the surface properties.

Our first-principle calculations clearly show that for each of the group VI elements examined, the passivation of the Ga-terminated $\mathrm{GaSb}(001)$ surface is thermodynamically favored over the passivation of the Sb-terminated GaSb surface. The oxygen adsorption on the Ga-terminated surface reduces the gap-region DOS significantly because the interactions of gallium surface bonds with the oxygen orbitals result in bonding and antibonding states in the deep valence band and conduction band. Indeed, it has been shown that the $\mathrm{Ga}_{2} \mathrm{O}_{3} / \mathrm{GaAs}$ interface formed by single source deposition of $\mathrm{Ga}_{2} \mathrm{O}_{3}$ on the clean $\mathrm{GaAs}(001)$ surface exhibited the improved electronic properties. ${ }^{19}$ On the other hand, the for- 
mation of $\mathrm{Sb}-\mathrm{O}$ bonds reduces the density of gap-region states only slightly. As the formation of elemental $\mathrm{Sb}$ rather than of antimony oxide is thermodynamically favored during the thermal oxidation of $\mathrm{GaSb},{ }^{2}$ the existence of elemental antimony along with $\mathrm{Sb}-\mathrm{O}$ bonds is presumed to be a primary cause for the high density of midgap states observed on the oxidized GaSb surface.

The successful passivation of GaAs surfaces by aqueous sulfide solutions relies not only on the formation of $\mathrm{Ga}-\mathrm{S}$ bonds, which modify the surface electronic structure, but also on the decrease in the amount of arsenic-related species, which are soluble in alkaline sulfide solutions. Antimony oxide, however, is insoluble in acidic or alkaline aqueous solutions. ${ }^{20}$ In addition, GaSb is much more reactive than GaAs. As a result, an aqueous processing leads to a high density of Ga-OH groups on the surface. ${ }^{21}$ The use of anhydrous benzene as a passivation medium can limit or eliminate the growth of such surface oxide. ${ }^{22}$ The increased PL intensity was consistently observed from the GaSb samples treated by $\mathrm{S}, \mathrm{Se}$, or Te in the nonaqueous medium. The enhancement in the photoluminescence indicates the decrease in the surface band bending or the surface recombination velocity, a consequence of the reduction of energy states within the band gap. While the motion of the surface Fermi energy has not been determined, the XPS analysis reveals the surface bonding states and chemical compositions resulting from each of the three chalcogen treatments.

The nonaqueous passivation regime employs the macrocyclic polyethers to solubilize the chalcogenide salts $\mathrm{Na}_{2} X$ ( $X=\mathrm{S}, \mathrm{Se}$, and $\mathrm{Te}$ ) into the nonpolar, aprotic benzene solvent. The generated "naked" chalcogenide anions are expected to be potent nucleophiles with respect to the GaSb surface. ${ }^{14}$ The relative reactivity of the chalcogenide anion is likely governed by its negative charge density, (charge radius ratio), which decreases from $\mathrm{S}^{2-}$ to $\mathrm{Te}^{2-}$. The binding-energy calculations indicate that the strength of the Ga- $X$ linkage decreases, as the size of the chalcogenide ion increases: the binding energy is highest for $\mathrm{Ga}-\mathrm{S}(3.49 \mathrm{eV})$ and lowest for $\mathrm{Ga}-\mathrm{Te}(2.38 \mathrm{eV})$, at a full monolayer coverage. These trends are reflected in the chemical compositions of the passivated surfaces (Table II): the concentration of Ga- $X$ specie is highest for $\mathrm{Ga}-\mathrm{S}$ compound, and decrease as $X$ varies from $\mathrm{S}$ to $\mathrm{Te}$.

The increase in the surface chalcogenide content during the passivation is accompanied by a loss of antimony. For example, as the processing time for the nonaqueous passivation of $\mathrm{GaSb}$ with $\mathrm{Na}_{2} \mathrm{Se}$ increases from a minute to an hour, the increase in the Se content (the Se/Ga XPS peak intensity ratio increases from 0.36 to 0.48 ) is paralleled by a decrease in the $\mathrm{Sb} / \mathrm{Ga}$ ratio (from 0.60 to 0.44 ). This is consistent with a form of anion exchange process, where the surface antimony is displaced and solubilized from GaSb by the chalcogenide ion. Therefore, the $\mathrm{Sb}-X(X=\mathrm{S}, \mathrm{Se}, \mathrm{Te})$ bond formation competes with the substitution of surface $\mathrm{Sb}$ by $X$.

\section{B. Adsorption Kinetics: Chemisorption of Se on the GaSb Surface}

In most cases, the chemisorption process occurring on a solid surface is mediated by a precursor state, ${ }^{23}$ and this model has been used successfully in analyzing the initial stage of oxidation of $\mathrm{Si}$ and GaAs surfaces. ${ }^{24,25}$ Applying such model in our case, Se anions in the solution would first physisorb on the GaSb surface, which is termed as an intermediate state or a precursor state. The weakly bonded Se ions can either desorb from the surface or form chemical bonds with surface atoms. Since little $\mathrm{Sb}-\mathrm{Se}$ was observed, $\mathrm{Ga}-\mathrm{Se}$ is considered to be the predominant stable state on the surface and its desorption into the solution is slow and negligible. The adsorption process is thus expressed as

$$
\mathrm{Se}_{\text {(solut) }}^{2-}+\underset{k_{d}}{\stackrel{\alpha}{\rightleftarrows}} \mathrm{Se}_{(\text {interm) }}^{*} \stackrel{k_{a}}{\longrightarrow} \mathrm{Se}-\mathrm{Ga},
$$

where $\alpha$ is the trapping probability and $k_{d}$ and $k_{a}$ are the rate constants of intermediated states desorbed into the solution or converted to the chemisorbed states.

Assuming that (1) the concentration of the intermediate state $\mathrm{Se}^{*}$ does not change with time, i.e., reaches a steady state; (2) the transfer from the precursor state to the chemisorbed state is the rate-determining step; and (3) the adsorption is the first-order reaction, the following rate equations are written:

$$
\begin{aligned}
& \frac{d C_{\mathrm{Se}^{*}}}{d t}=0 \Rightarrow \alpha C_{\mathrm{sol}} C_{\mathrm{empty}}-\left(k_{d}+k_{a}\right) C_{\mathrm{Se}^{*}}=0 \\
& r=\frac{d C}{d t}=k_{a} C_{\mathrm{Se}^{*}} .
\end{aligned}
$$

In these equations, the concentration is defined as the atomic ratio of surface specie to the substrate $\mathrm{Ga}$ atoms. $C_{\mathrm{empty}}, C_{\mathrm{Se}^{*} \text {, }}$ and $C$ represent the concentrations of empty sites, the intermediate state $\mathrm{Se}^{*}$, and the $\mathrm{Se}-\mathrm{Ga}$ chemical-bonded states on the surface, respectively. $C_{\text {sol }}$ is the concentration of $\mathrm{Na}_{2} \mathrm{Se}$ in the benzene-based solution.

The chemisorption of $\mathrm{Se}$ at room temperature using a benzene-based saturated $\mathrm{Na}_{2} \mathrm{Se}$ solution results in less than two monolayers coverage of surface selenide after an hour of passivation treatment. Any diffusional process, either in the liquid phase or through the developed passivation layer, is therefore neglected in describing the surface passivation layer formation, and the formation of $\mathrm{Se}-\mathrm{Ga}$ chemical bonds is considered to be the slowest reaction step in the model. The passivation process is analyzed on the basis of a single precursor-mediated chemisorption, and the kinetics is derived in two distinctive cases, the Langmuir-based adsorption and coverage-dependent adsorption.

\section{Langmuir-based Adsorption}

Langmuir-based adsorptiom is a simple but a useful model in the analysis of chemical kinetic data. In this model, the total number of available adsorption sites on the surface is fixed, and the adsorption energy is considered to be the site and coverage independent.

Site conservation requires that

$$
N_{\text {empty }}+N_{\mathrm{Se}^{*}}+N=N_{T} \text {, }
$$

where $N_{T}$ is the density of the total available sites. Dividing both sides of Eq. (3) by the site density of Ga results in 


$$
C_{\text {empty }}+C_{\mathrm{Se}^{*}}+C=C_{T},
$$

where $C_{T}$ the saturation value at a maximum chemisorption. Combining Eqs. (1), (2), and (4), the concentration of the chemisorbed Se as a function of the processing time is given by

$$
\begin{aligned}
& C=C_{T}\left(1-e^{-A t}\right) \\
& A=\frac{\alpha C_{\mathrm{sol}} K_{a}}{\alpha C_{\mathrm{sol}}+k_{a}+k_{d}} .
\end{aligned}
$$

Based on the work of Seah and Dench ${ }^{26}$ the mean free path of electrons emitted from Ga2 $p$ core level is approximately $1.3 \mathrm{~nm}$. Given that the sampling volume is much greater than a monolayer, it is therefore assumed that the detected gallium by the XPS measurement is constant. The measured data set of Se-to-Ga XPS intensity ratio versus processing time was then used for the parametric fit of the Langmuir-based adsorption model in Eq. (5), and the optimal fit parameters obtained are as follows $C_{T}=0.48 \pm 0.03$ and $A=0.029 \pm 0.008$. As the Se concentration is close to the saturation level, the adsorption rate is remarkably reduced due to the short available adsorption sites.

\section{Coverage-Dependent Adsorption}

The Langmuir-based adsorption model is valid in the case of a perfectly uniform surface with noninteracting adatoms. In practice, the adatom/substrate system may exhibit a coverage-dependent adsorption behavior, and the extent of this behavior depends on the specific adatom interactions. An alternative model, without assuming a limiting or saturating coverage, is derived on the basis of a coverage-dependent activation energy. The Se coverage obtained under the preparation condition in this study is assumed to be far from the saturation level, and the reduced adsorption rate with Se coverage is ascribed to an increased, coverage-dependent activation barrier. In the simplest embodiment, the activation energy linearly increases with the surface Se concentration, and the rate constant $k_{a}$ is thus given by

$$
\begin{aligned}
& k_{a}=\nu_{a} \exp \left[-\left(\frac{E_{a}^{0}+a C}{k_{B} T}\right)\right]=k_{a}^{0} \exp \left(-\frac{a C}{k_{B} T}\right) \\
& k_{a}^{0}=\nu_{a} \exp \left[-\left(\frac{E_{a}^{0}}{k_{B} T}\right)\right],
\end{aligned}
$$

where $k_{B}$ is the Boltzmann's constant and $\nu_{a}$ is the frequency factor. $E_{a}^{0}$ and $k_{a}^{0}$ are the initial energy barrier and the corresponding rate constant at the limit of a zero coverage, respectively.

From Eqs. (1), (2), and (7), the concentration of surface selenide is derived as

$$
C=\frac{k_{B} T}{a} \ln \left(1+\frac{a k_{a}^{0} C_{\mathrm{Se}^{*}}}{k_{B} T} t\right) .
$$

The least square fit of the experimental points to the previous model is given in Fig. 4. The derived fitting parameters are the following:

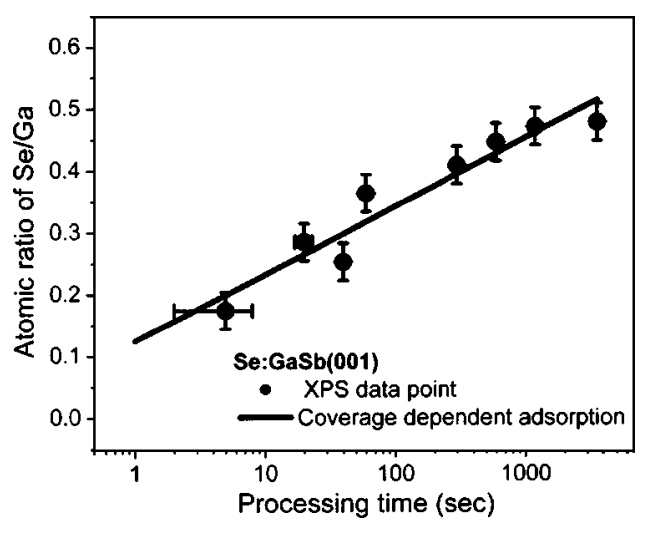

FIG. 4. Evolution of the surface selenide content vs the processing time. The solid dots are the atomic ratio of Se-to-Ga, derived from the XPS peak intensity ratio of $\mathrm{Se}$ and $\mathrm{Ga}$, with each processing time. The line is the least square fit of the kinetic data to the coverage-dependent adsorption model in Eq. (9) at a $95 \%$ confidence limit.

$$
a=0.54 \pm 0.05 \mathrm{eV}, \quad \mathrm{k}_{\mathrm{a}}=k_{a}^{0} \exp \left(-\frac{0.54 \mathrm{eV}}{k_{B} T} \times C\right),
$$

and the initial adsorption rate is $r^{0}=k_{a}^{0} C_{\mathrm{Se}^{*}}=0.60 \pm 0.24 \mathrm{~s}^{-1}$ at the limit of a zero coverage using a $95 \%$ confidence limit.

The activation energy is the energy required to enable the reaction to proceed, whereas the binding energy represents the potential well due to the adatom bound to the surface. Both energies can be surface-coverage dependent. As the adatom coverage increases, it becomes energetically more difficult for adsorption, and the surface bond energy is reduced due to the increased repulsive force between adatoms.

The coverage-dependent component of the activation energy $\left(E_{a}(C)-E_{a}(0)\right)$ as a function of Se-to-Ga atomic ratio $(C)$ is plotted in Fig. 5. The conversion of the XPS intensity ratio of Se-to-Ga to the Se coverage $(\theta)$, in the units of a monolayer, is based on the surface layer model, ${ }^{27}$ which correlates the attenuation of the XPS intensity of the substrate atom with the overlayer thickness. Using a value of $1.3 \mathrm{~nm}$ as the mean free path of electrons emitted from $\mathrm{Ga} 2 p$ core level with the takeoff angle of $75^{\circ}$, the $\mathrm{Se} / \mathrm{Ga}$ atomic ratio of 0.26 is achieved at a full single monolayer coverage on the

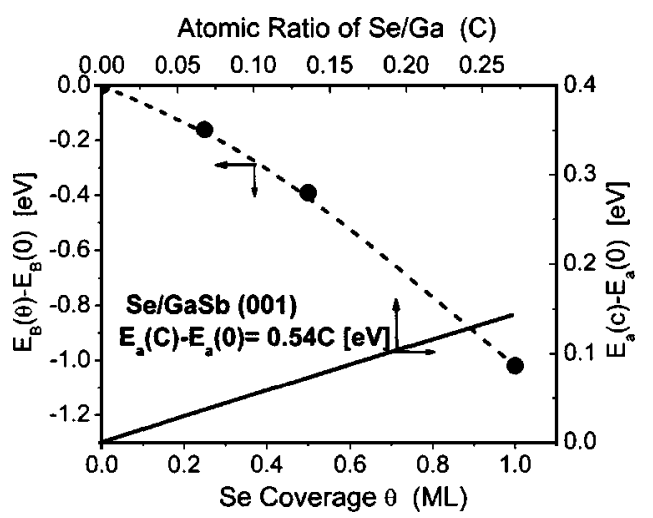

FIG. 5. Variation of the activation energy and $\mathrm{Se}-/ \mathrm{Ga}$ binding energy as a function of Se coverage. Solid line is a plot of the increase in the activation energy $\left(E_{a}(C)-E_{a}(0)\right)$ vs atomic ratio of Se Ga (C) using the determined fitting parameter. Dashed line is a plot of the change of $\mathrm{Ga}-\mathrm{Se}$ binding energy $\left(E_{B}(\theta)-E_{B}(0)\right)$ as the Se coverage $(\theta)$ increases to a monolayer. 
$\mathrm{GaSb}(001)$ surface. As see in Fig. 5, from zero to a full monolayer coverage, the activation energy increases by an amount of $\sim 0.14 \pm 0.02 \mathrm{eV}$. The change in the Se-Ga binding energy $\left(E_{B}(\theta)-E_{B}(0)\right)$ as a function of the Se coverage was determined from the first principles calculations, which results for $\theta_{\mathrm{se}}=1 / 4,1 / 2$ and $1 \mathrm{ML}$, and is also shown in Fig. 5. Over the initial stage of the adsorption, the Se-Ga binding energy reduces by $\sim 1 \mathrm{eV}$ going from zero to a full single monolayer coverage. Given the trends exhibited by the experimental data as well as these calculated results, the coverage-dependent model appears to offer a more physical picture in describing the Se adsorption on the GaSb surface in the initial stage.

\section{CONCLUSIONS}

The electronic properties and chemical structure of the $n$-GaSb surface adsorbed with chalcogen atoms were investigated. The results of the surface electronic structure using a discrete Fourier transform-based calculations, indicate that the adsorption of chalcogen atoms on the Ga-terminated GaSb surface significantly reduces the density of gap-region surface states. PL measurements imply that the electronic passivation efficiency is dependent on both the chalcogenide used and the specific reaction medium applied. The nonaqueous chalcogenide treatment using $\mathrm{Na}_{2} \mathrm{~S}, \mathrm{Na}_{2} \mathrm{Se}, \mathrm{Na}_{2} \mathrm{Te}$ resulted in up to an eightfold PL enhancement. Surface chemistry analysis suggests that the adsorption of $\mathrm{S}$ on the $\mathrm{GaSb}$ surface is more favorable than that of the larger and less reactive chalcogen $\mathrm{Se}$ and $\mathrm{Te}$, and the incorporation of heavier chalcogen atoms in the GaSb surface most likely proceeds by displacement of the surface antimony. The initial adsorption of $\mathrm{Se}$ on the GsSb surface proceeds rapidly, followed by a remarkably reduced adsorption rate as the selenide coverage increases. The adsorption process is analyzed on the basis of a single precursor-mediated chemisorption.

\section{ACKNOWLEDGMENT}

This work was funded by the Army Research Office, the DARPA, and the NSF-funded UW-MRSEC. Also, the com- puter time was granted by the DOD High-Performance Computing Moderization Programs at the Engineer Research and Development Center and the Naval Oceanographic Office.

${ }^{1}$ A. G. Milnes and A. Y. Ployakov, Solid-State Electron. 36, 803 (1993).

${ }^{2}$ G. P. Schwartz, G. J. Gualtieri, J. E. Griffiths, C. D. Thurmond, and B. Schwartz, J. Electrochem. Soc. 127, 2488 (1980).

${ }^{3}$ Z. Lu, Y. Jiang, W. I. Wang, M. C. Teich, and R. M. Osgood Jr., J. Vac. Sci. Technol. B 10, 1856 (1992).

${ }^{4}$ Z. Y. Liu, B. Hawkins, and T. F. Kuech, J. Vac. Sci. Technol. B 21, 71 (2003).

${ }^{5}$ M. S. Carpenter, M. R. Melloch, and T. E. Dungan, Appl. Phys. Lett. 53, 66 (1988).

${ }^{6}$ M. Perotin, P. Coudray, L. Gouskov, H. Luquet, C. Llinares, J. J. Bonnet, L. Soonckindt, and B. Lambert, J. Electron. Mater. 23, 7 (1994).

${ }^{7}$ Beena Annie Kuruvilla, A. Datta, G. S. Shekhawat, A. K. Sharma, P. D. Vyas, R. P. Gupta, and S. K. Kulkarni, J. Appl. Phys. 80, 6274 (1996).

${ }^{8}$ C. J. Sandroff, R. N. Nottenburg, J.-C Bischoff, and R. Bhat, Appl. Phys. Lett. 51, 33 (1987).

${ }^{9}$ S. A. Chambers and V. S. Sundaram, Appl. Phys. Lett. 57, 2342 (1990).

${ }^{10}$ A. M. Green and W. E. Spicer, J. Vac. Sci. Technol. A 11, 1061 (1993).

${ }^{11}$ T. Ohno, Surf. Sci. 255, 229 (1991).

${ }^{12}$ D. K. Biegelsen, R. D. Bringans, J. E. Northrup, and L.-E. Swartz, Phys. Rev. B 49, 5424 (1994).

${ }^{13}$ J. X. Sun, D. J. Seo, W. L. O'Brien, F. J. Himpsel, A. B. Ellis, and T. F. Kuech, J. Appl. Phys. 85, 969 (1999).

${ }^{14}$ Z. Y. Liu, D. A. Saulys, and T. F. Kuech, Appl. Phys. Lett. 83, 2587 (2003).

${ }^{15}$ J. P. Perdew, J. A. Chevary, S. H. Vosko, K. A. Jackson, M. R. Pederson, D. J. Singh, and C. Fiolhais, Phys. Rev. B 46, 6671 (1992).

${ }^{16}$ J. A. White, D. M. Bird, M. C. Payne, and I. Stich, Phys. Rev. Lett. 73, 1404 (1994).

${ }^{17}$ D. B. Wittry and D. F. Kyser, J. Appl. Phys. 38, 375 (1967).

${ }^{18}$ K. Mettler, Appl. Phys. 12, 75 (1977).

${ }^{19}$ M. Passlack, M. Hong, J. P. Mannaerts, R. L. Opila, and F. Ren, Appl. Phys. Lett. 69, 302 (1996).

${ }^{20} \mathrm{~J}$. Hunsberger, in Handbook of Chemistry, Physics and Electrochemical Series, edited by R. C. Weast (CRC, Cleveland, 1974).

${ }^{21}$ C. Webb and M. Lichtensteiger, J. Vac. Sci. Technol. 21, 659 (1982).

${ }^{22}$ Z. Y. Liu, D. A. Saulys, and T. F. Kuech, Appl. Phys. Lett. 83, 2587 (2003).

${ }^{23}$ W. H. Weinberg, in Kinetics of Interface Reactions, edited by N. Grunze and H. J. Kreuzer (Springer-Verlag, New York, 1987), Vol. 8, p. 94.

${ }^{24}$ A. Stockhausen, T. U. Kampen, and W. Monch, Appl. Surf. Sci. 56-58, 795 (1992)

${ }^{25}$ W. Monch, Surf. Sci. 168, 577 (1986).

${ }^{26}$ M. P. Seah and W. A. Dench, Surf. Interface Anal. 1, 2 (1979).

${ }^{27}$ M. P. Seah, in Practical Surface Analysis: Auger and X-ray Photoelectron Spectroscopy, edited by D. Briggs and M. P. Seah (Wiley, New York, 1990), Vol. 1, p. 201. 